\title{
Alert to a European epidemic
}

\section{Funds must be forthcoming for an effective EU Centre for Disease Control.}

\section{S. Ragnar Norrby}

The eruption of severe acute respiratory syndrome (SARS) in 2003 was characterized by the rapid spread of the outbreak across several countries around the world. Although the World Health Organization (WHO) provided extremely fast information and support to afflicted countries, the European network of state epidemiologists and national agencies failed to respond quickly enough. Important information about possible cases of SARS or European travel restrictions were often communicated with delays of 48 hours or more. European healthcare officials hope to avoid repeats of such inadequate responses, and are determined that faster coordination and better surveillance will be made possible by a European Centre for Disease Control (ECDC), similar to the US Centers for Disease Control and Prevention (CDC) in Atlanta, Georgia. A decision about the new ECDC director is expected shortly, but it is already clear that this director will face an uphill struggle to create a European equivalent to the CDC.

Uncontrollable outbreaks of infectious diseases are a public-health crisis waiting to happen. New and emerging infections, including SARS and HIV/AIDS have appeared, and older infections, such as tuberculosis and malaria, are far from being eliminated. Resistance to antibiotics continues to increase, with no new drugs on the horizon. We also face threats from large-scale outbreaks of infectious diseases, especially an influenza pandemic, and from the deliberate release of infectious agents by criminals or terrorist groups. Few of these problems are being addressed in a constructive way industry is failing to develop new antibiotics and vaccine production is insufficient to meet the needs of a pandemic.

Against this background, it seems clear that Europe would benefit from a regional organization similar to the Atlanta CDC, which has large resources in terms of scientific expertise and laboratory capacity, and which can deploy field forces (epidemiologists and laboratory equipment) to the site of an outbreak at very short notice. The current difficulties of the European Union in collating and disseminating epidemiological information - as seen with SARS — will only get worse with the addition of ten new member states. Therefore the creation of an independent agency for surveillance and control of communicable diseases has been welcomed, and from 2005 the ECDC will operate from a base in Stockholm, Sweden.

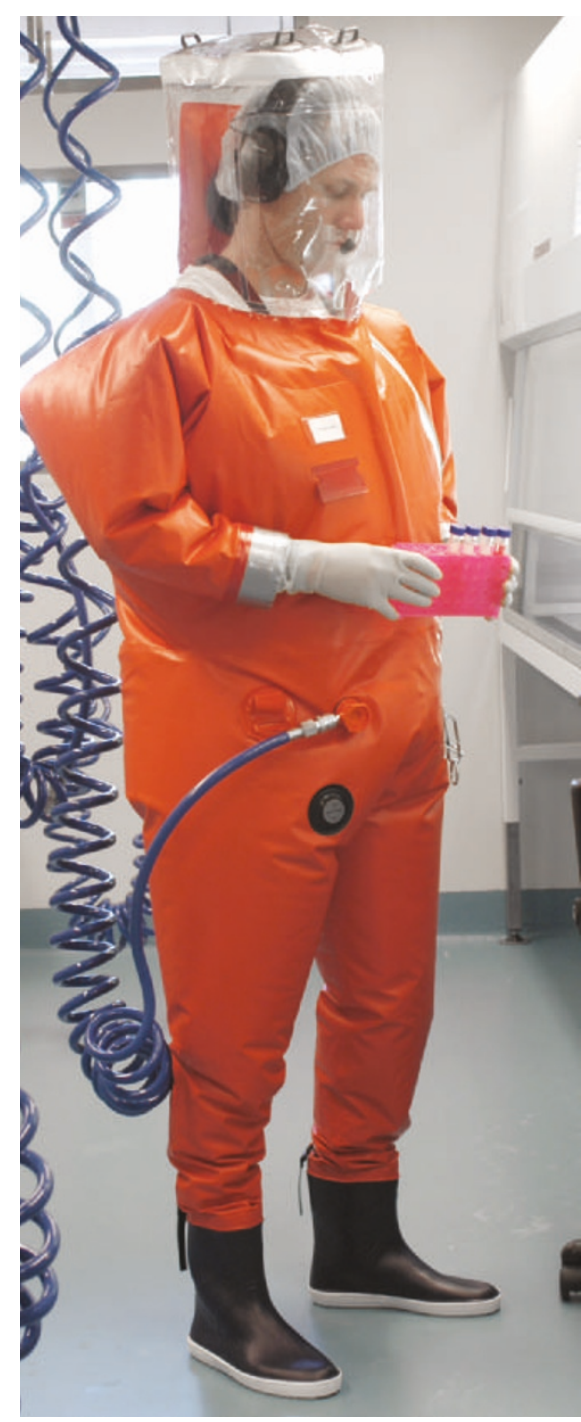

Staff trained in lab work are in short supply.

But there remain fears that the ECDC has been structured in a way that leaves it powerless to effect change. It will lack both regulatory authority and laboratory resources which will curtail its independence. Lab resources are considered by many to be a necessity for modern infectious diseases epidemiology. But this particular omission could be corrected if the third weakness, a small budget, is improved over time.

Why does Europe need its own CDC? On a global level, the WHO regularly communicates information from official government sources. But such information is not always complete, as governments try to balance openness with the negative effects that epidemics have on travel and tourism. In addition to the CDC - which tracks both the US and global situations - there is the internet-based ProMed, now ten years old. This e-mail system (open to anyone who is interested) is run by the International Society for Infectious Diseases and is a major source of rapid and reliable information. One advantage of the Atlanta CDC is its ability to combine surveillance with active field forces. At present, Europeans have to call the CDC or the WHO for help when there are outbreaks in Europe. Although SARS was stopped in its tracks by such global coordination, Europe may not be solucky next time.

One of the main tasks of the ECDC is to "search for, collect, collate, evaluate and disseminate relevant scientific and technical data"'. This is currently carried out on a national level in Europe. But EU countries vary considerably in terms of resources and which diseases are notifiable. The surveillance systems and the quality of the data generated also vary. For example, each year more cases of salmonellosis are reported for Swedish tourists visiting some European countries than for the entire native populations of those countries. Standardization of these systems is not likely to occur in the near future, except for the most severe infections, which will be regulated by the new International Health Regulations, to be ratified by the WHO in 2005 .

Modern epidemiological investigations go beyond collection and analysis of clinical reports, often requiring sophisticated laboratory studies of the microorganisms molecular epidemiology - to achieve reliable analyses. Accordingly, CDC and several European government agencies are given access to large laboratory facilities. But the ECDC's preliminary budget for 2005-2007 does not include funds for lab activities within the centre itself, nor will there be sufficient funds to pay for more than limited services at national laboratories.

Widespread outbreaks of communicable diseases also require access to epidemiological expertise that can be rapidly deployed in the field. Containment of the 2003 SARS outbreak was principally due to the WHO's rapid deployment of advisers to manage the epidemic on site. The WHO and CDC have also helped control many other outbreaks, including Ebola in Africa. Europe lacks coordinated resources of this kind.

Together, the relatively small ECDC budget and lack of facilities will prohibit the creation and maintenance of a European field force. They will also limit the independence of the centre and will make it harder to recruit the most competent staff. In the short term, locating the ECDC close to existing facilities in Stockholm may improve this 


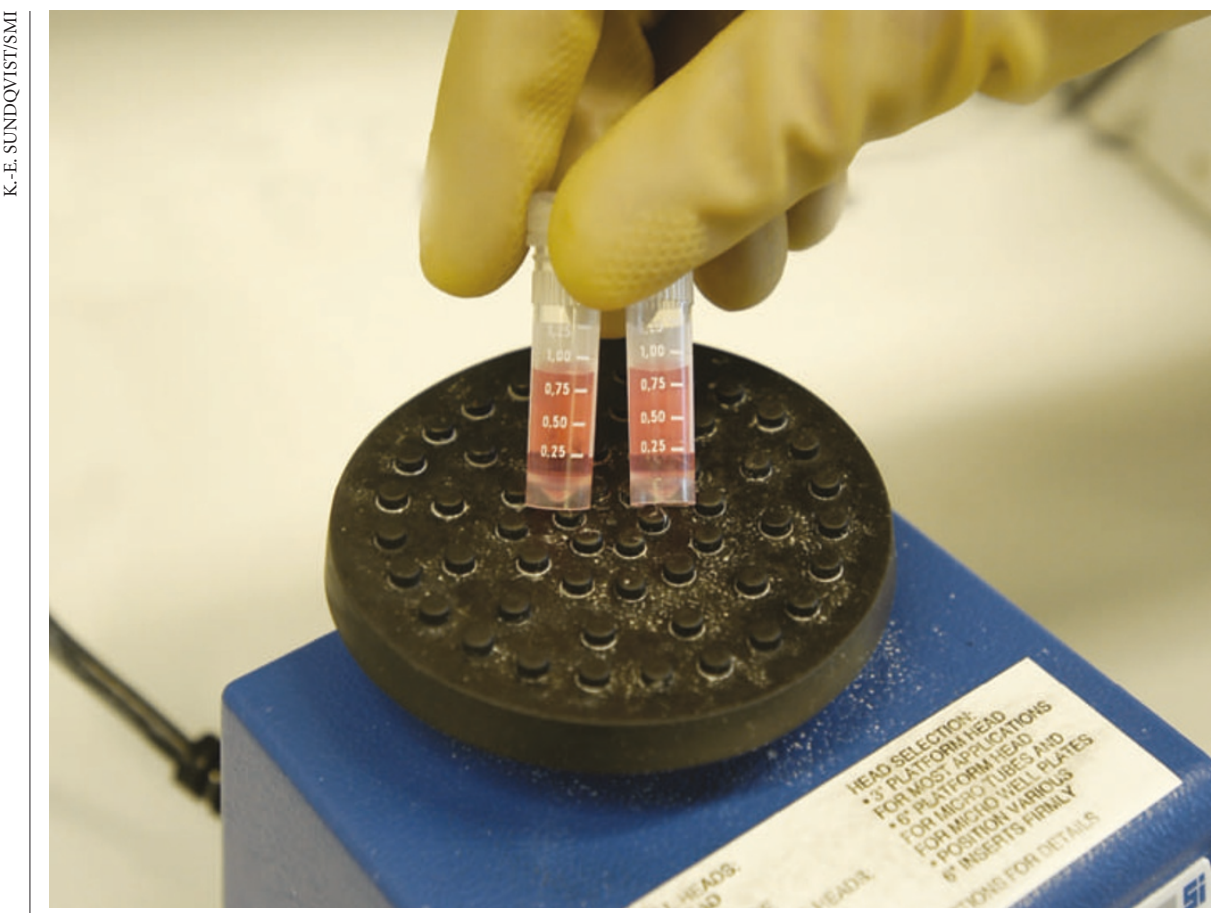

Sweden has one of the few labs equipped to deal with the hazards of handling infectious diseases.

situation. In addition to the excellent facilities at the Karolinska Institute, the Swedish Institute for Infectious Disease Control has an advanced biosecurity (biosafety level 4, BSL4) laboratory. The new ECDC director should be able to allocate some funds for lab work, subject to approval by the management board.

In 2002, Sweden formed a national field force consisting of 26 physicians, veterinarians, nurses and health inspectors - which to my knowledge is the only one of its kind in Europe. They have already assisted the WHO during the SARS outbreak and in investigations of avian flu in Asia. But this group has only rudimentary training in lab work, and so for European outbreaks the affected countries must rely on local facilities or laboratory services organized by the WHO or offered by the CDC. Without its own lab resources, the ECDC cannot hope to fill this gap.

What ECDC can, and hopefully will, do is train members of an international task force and coordinate their activities within or outside Europe. Because of its small allocated budget, salaries for such staff members can be covered by ECDC only when an outbreak occurs. Between outbreaks, members of the force must be supported by their home countries.

\section{Need for a leader}

The ECDC will have no regulatory power ${ }^{1}$ in accordance with the rule that the European Union does not issue directives in the field of public health. This is why the choice of director is so important. The director must be a leader who can attract epidemiologists, microbiologists and scientists of very high standards. The tasks of the ECDC are formulated in such a general way that the board and the director will have considerable freedom to develop a working plan for the centre. But without the right sort of leadership, ECDC will struggle to be effective.

One area where Europe desperately needs coordinated actions is in the use and abuse of antibiotic drugs. There are striking differences in antibiotic consumption between European countries, which cannot be explained by the burden of disease $e^{2}$. For example, France prescribes about ten times more antibiotics than The Netherlands, although it is clear that the French do not suffer ten times more infectious diseases. Overuse is one reason for growing resistance to these drugs.

The problems surrounding lack of new antibiotics - we are close to the point where we might return to the pre-antibiotic era ${ }^{3}$. In addition to greater morbidity and mortality, resistance increases costs through prolonged hospital stays and the use of more costly drugs.

What can the ECDC contribute to the fight against resistance? It could expand surveillance programmes to include cases of infections caused by resistant organisms, and promote campaigns to reduce national differences in antibiotic consumption. By applying political pressure, the ECDC could reinforce the EU policy that limits access to antimicrobial drugs to prescription alone. At present it is possible to buy antibiotics over the counter in many European countries.

One of the most worrying threats is a new influenza pandemic, whose likelihood increases with every outbreak of avian flu in Southeast Asia and Europe. Of particular resistance are heightened by a concern is that the transmission of avian flu in Asia has led to high death rates among bird handlers - although the viruses have not been transmissible between humans. If even one of them becomes transmissible, we could be faced with an outbreak similar to the 'Spanish flu' of 1918. That epidemic resulted in approximately 40 million deaths and many who died were young and previously healthy individuals. In Europe, and elsewhere, the amount of influenza vaccine produced would be insufficient to prevent such a scenario. The ECDC needs to issue guidelines for member states, facilitate vaccine availability and support the development of national plans for a pandemic.

\section{Forward thinking}

In addition, the ECDC could help the European Union prepare for possible deliberate releases of microorganisms. At present, the European response is handled through the Directorate General for Health and Consumer Protection (DG SANCO) in Luxemburg, but that responsibility (and associated funds) should be taken over by ECDC. There are also plans to increase the number of BSL4 laboratories in Europe, from the handful in France, Germany, Britain and Sweden. It would make sense for the ECDC to form a network between these existing laboratories - thereby avoiding redundancy of effort. If you consider the expense of building these complex facilities (in excess of US\$15 million each) and the type of rare infections they study, the money allocated to planned facilities in Germany and The Netherlands would be better spent elsewhere.

Clearly, the ECDC cannot hope to become an equivalent to the CDC in the short term. The lack of authority and lab facilities will hamper ECDC's ability to become an efficient coordinating force for infectious disease control in Europe. And the ECDC will find it hard to train, finance and deploy an epidemiological field force within its allocated budget. But with the expectation of future funding increases, the ECDC should initiate field forces within the member states. A substantial boost to the ECDC would be money well spent as the costs of antibiotic resistance, let alone of SARS or influenza outbreaks, are likely to be enormous.

S. Ragnar Norrby is at the Swedish Institute for Infectious Disease Control, Se 17182

Solna, Sweden.

1. Cox, P., Roche, D. R. Official Journal of the European Union L142: 1-11 (2004).

2. Cars, O., Mölstad, S., Melander, A. Lancet 357, 1851-1853 (2001). 3. Shlaes, D. M., Projan, S. J. \& Edwards Jr, J. E. American Society of Microbiology News 2004 70, 275-281 (2004).

Competing interest: The author is director-general of the Swedish Institute for Infectious Disease Control, an expert government agency for surveillance, control and research in the field of communicable diseases. Citizens from the host country are excluded from the ECDC director position. 